\title{
Antibodies at the Center of Immunotherapy: Commentary on "Moving a Carbohydrate Mimetic Peptide into the Clinic"
}

Issam Makhoul, Bejatohlah Monzavi-Karbassi and Thomas Kieber-Emmons*

Winthrop P. Rockefeller Cancer Institute, University of Arkansas for Medical Sciences, Little Rock Ar. 72205, USA

*Corresponding Author: Kieber-Emmons T, Winthrop P. Rockefeller Cancer Institute, University of Arkansas for Medical Sciences, Little Rock Ar-72205, USA, Tel: 501-526-5930; E-mail: tke@uams.edu

Received date: October 17, 2016; Accepted date: October 21, 2016; Published date: October 26, 2016

Copyright: @ 2016 Makhoul I, et al. This is an open-access article distributed under the terms of the Creative Commons Attribution License, which permits unrestricted use, distribution, and reproduction in any medium, provided the original author and source are credited.

\section{Commentary}

The idea that we can activate the immune system to fight cancer is starting to pay off in clinical practice. Instead of targeting the tumor itself, as in conventional cancer therapies, activating and mobilizing the immune system to attack its "abnormal self" necessitates the training of the immune system to see a collection of antigens expressed on tumor cells. We have recently shown that a novel immunogen can recruit antibodies to breast cancer tumors that can inhibit tumor cell growth [1]. These vaccine-induced antibodies are cross-reactive with Tumor Associated Carbohydrate Antigens (TACA), which are intimately involved in cell survival signaling. The ability of these antibodies to bind to TACA and interfere with signalling events also suggest additive or synergistic modality with chemotherapeutics that are cytotoxic. Most importantly, our studies suggested a potential clinical benefit to the combination of TACA-reactive antibodies as illustrated by the multiple antibodies induced with the P10s vaccine in cancer patients [1]. The sensitization of cancer cell via inhibition of cell survival pathways, show for the first time that signalling pathways interrupted by vaccine induced TACA reactive antibodies causes tumor regression in combination with standard of care chemotherapeutics.

Progress in our ability to manipulate the immune system led to the discovery of monoclonal antibodies (mAbs) that are specific to one epitope of an antigen. MAbs produced ex-vivo are fulfilling their original promise as pharmaceuticals in cancer treatment [2-5] and, as investigational tools, by helping investigators fine tune their manipulations of the immune system and design other immune targeted therapies. Our experience with the development of an antiglycan vaccine allowed us to move the process of producing antiglycan MAbs from the ex-vivo to the in-vivo setting and to be able to induce the production of multiple mAbs in patients using the same therapeutic agents, our $\mathrm{P} 10$ vaccine.

Many novel mAbs continue to enter the clinic, each designed with modifications to structure aimed at further improving efficacy [6,7]. As we move into an era of precision and personalized medicine, it will become increasingly important to develop closer links between emerging mechanistic insights, mediated by tumor associated antigens (TAA), and the clinical development of mAbs. Glycans or TACA are among TAA targeted by mAbs [8,9]. The ideal target of cancer therapeutics would be a molecule(s) critical for tumor cell survival, expressed at elevated levels on the tumor cell surface and therapeutic benefit should be demonstrable with antibody or $\mathrm{T}$ cells to this molecule. By these criteria, TACA stand out as excellent targeted candidates. TACA are intimately related with pathways that mediate cell survival. Tumors expressing a high level of certain types of TACAs exhibit greater metastasis and progression as reflected in decreased patient survival rate than those expressing low level of these TACAs, [10]. mAbs targeting glycans that are either in the clinic or under preclinical development include those for GD2 [7,10-14], and tumorassociated Lewis (Le) glycans $[15,16]$.

TACA, which are elevated in a wide range of solid tumors, mediate apoptosis in tumor cells while sparing normal cells, demonstrating both selectivity and therapeutic activity. When considering this family of antigens as targets, the first challenge is whether mAbs will trigger apoptotic signals because many receptor binding antibodies block, rather than trigger signals. While immunotherapy has focused on approval of mAbs for passive therapy, there are parallel efforts to develop immunogens to induce sustained antibody mediated immunity against TACA [17-20].

Our experience with cancer antigens suggests that TACA are panantigens shared across tumor types and are significantly different from the ones expressed on normal cells. TACA are pan-targets because they are intimately involved in cell signalling pathways associated with all cancer cells. The discovery of glycan pan-antigens led to a concept of a pan-approach to immune therapy of cancer where all cancers are targeted by one treatment designed by special manipulation of the immune system. This concept suggests approaches to induce and maintain an immune response against multiple TACA, thus unleashing a powerful multi-prong attack against a tumor. In this context multivalent forms of TACA-based vaccines in particular are meant to induce responses across multiple TACAs by inducing subpopulations of antibodies reactive with the constitutive components of the multivalent vaccine [21-23].

In contrast to TACA-based vaccines, we have developed potential vaccines based on carbohydrate-mimetic peptides (CMPs) [24]. This approach is similar to using anti-idiotypic antibodies as mimics of TACAs $[9,25]$. We have shown that CMPs induce anti-tumor-reactive humoral [26-28] and cellular responses [29,30]. We have moved one of these CMPs, with the sequence WRYTAPVHLGDG (referred to as P10s) conjugated to the Pan-T-cell epitope PADRE, into an early-phase clinical trial in Stage IV breast-cancer subjects [1]. This CMP was designed to mimic and induce responses to TACAs that are associated with glycolipid moieties including the ganglioside GD2 and the LeY $[28,31]$.

P10s was computer designed to react with the anti-GD2 antibody ME36.1 and the anti-LeY antibody BR55-2 [28,32]. Therefore, we have extended the notion of mimicry by considering CMPs as panimmunogens, inducing multiple sets of antibodies reactive with multiple TACAs when immunizing with a single agent [24-29,32]. Conceptually, CMP-induced responses might be a way to manipulate the immune system to generate beneficial low-affinity antibodies that 
would sidestep a variety of potential side effects from generating highaffinity antibodies to a particular TACA $[28,33]$ such as GD2 [34].

In keeping with the concept of being a pan-immunogen, P10s was found to react with several anti-GD2 MAbs that include 3F8 [35], 14.G2a [36] and ME36.1 [37] along with the anti-LeY mAb BR55-2 $[15,38]$. Unlike the other two anti-GD2 MAb 3F8 and 14.G2a, ME36.1 cross-reacts with GD3 [37]. Notably, in a recent project for prioritization of cancer antigens, 4 of the 75 selected antigens were gangliosides (GD2, GD3, fucosyl-GM1, and N-acetyl GM3), and additional targets, like the EGFR and the VEGFR, are known to interact with gangliosides $[39,40]$.

Our observation that $\mathrm{P} 10$ s immunization enhances reactivity to the ganglioside GD2 and LeY in humans is one example of inducing responses to multiple TACAs with a pan-immunogen, which has therapeutic ramifications such as driving epitope spreading. In our early phase clinical trial we observed some direct clinical benefit in one of our subjects with metastatic lesions as evaluated before and after vaccine treatment [1]. Inducing antibodies to multiple TACA can contribute to overcome immune-escape mechanisms since TACA are always expressed on tumor cells which increases the therapeutic potential of this cancer vaccine.

The evidence in this study further serves as a proof-of-principle for immunization using P10s-PADRE to induce TACA reactive antibodies that are proapoptotic. Monoclonal antibodies, such as anti-GD2 and LeY antibodies, can mediate signaling pathways extracellularly. This might be accomplished whereby apoptosis signals are transduced via reduction in the phosphorylation levels of focal adhesion kinase (FAK) and the activation of a MAPK family member, p38, upon the antibody binding. Knock down of FAK results in apoptosis and p38 activation. In this context $\mathrm{mAbs}$ have defined mechanistic pathways linked to their therapeutic function. Of significance then is that the P10s vaccine can induce antibodies with functionalities similar to mAbs reactive with GD2 and LeY and through these mechanistic pathways can function in association with chemotherapeutics to kill tumor cells or can sensitize tumor cells for more efficient tumor cell killing. This sensitization concept has now provided an opportunity to test the p10s vaccine in a phase 2 study involving HER 2 negative, ER positive breast cancer patients in the neoadjuvant setting (NCT02229084, http:// www.aymag.com/promising-breast-cancer-vaccine-clinical-trial-offeredarkansas/)

\section{Acknowledgements}

This work was supported by a Clinical Translational Award from the Department of Defense Breast Cancer Program (W81XWH-06-1-0542) to TKE. Also supported by the UAMS Translational Research Institute (TRI), UL1TR000039 through the NIH National Center for Research Resources and National Center for Advancing Translational Sciences and the UAMS Center for Microbial Pathogenesis and Host Inflammatory Responses, P20 GM103625. The content is solely the responsibility of the authors and does not necessarily represent the official views of the NIH or UAMS. Drs. Kieber-Emmons, Monzavi-Karbassi, and UAMS have a financial interest in the technology discussed in this publication. These financial interests have been reviewed and approved in accordance with the UAMS conflict of interest policies.

\section{References}

1. Makhoul I, Hutchins L, Emanuel PD, Pennisi A, Siegel E, et al. (2015) Moving a Carbohydrate Mimetic Peptide into the clinic. Hum Vaccin Immunother 11: 37-44.

2. Nadler LM, Stashenko P, Hardy R, Kaplan WD, Button LN, et al. (1980) Serotherapy of a patient with a monoclonal antibody directed against a human lymphoma-associated antigen. Cancer Res 40: 3147-3154.

3. Reichert JM, Rosensweig CJ, Faden LB, Dewitz MC (2005) Monoclonal antibody successes in the clinic. Nat Biotechnol 23: 1073-1078.

4. Steplewski Z, Thurin M, Kieber-Emmons T (2015) Antibodies: At The Nexus of Antigens and Cancer Vaccines. J Infect Dis 212: S59-s66.

5. Weiner GJ (2015) Building better monoclonal antibody-based therapeutics. Nat Rev Cancer 15: 361-370.

6. Li Q, Yi L, Marek P (2014) Antibodies and their multivalent constructs for cancer therapy. Protein Pept Lett 21: 1017-1024.

7. Navid F, Sondel PM, Barfield R, Shulkin BL, Kaufman RA, et al. (2014) Phase I trial of a novel anti-GD2 monoclonal antibody, Hu14.18K322A, designed to decrease toxicity in children with refractory or recurrent neuroblastoma. J Clin Oncol 32: 1445-1452.

8. Livingston PO, Hood C, Krug LM, Warren N, Kris MG, et al. (2005) Selection of GM2, fucosyl GM1, globo $\mathrm{H}$ and polysialic acid as targets on small cell lung cancers for antibody mediated immunotherapy. Cancer Immunol Immunother. 54: 1018-1025.

9. Rabu C, Mcintosh R, Jurasova Z, Durrant L (2012) Glycans as targets for therapeutic antitumor antibodies. Future Oncol. 8: 943-960.

10. Hakomori S (2001) Tumor-associated carbohydrate antigens defining tumor malignancy: basis for development of anti-cancer vaccines. Adv Exp Med Biol 491: 369-402.

11. Grant SC, Kostakoglu L, Kris MG, Yeh SD, Larson SM, et al. (1996). Targeting of small-cell lung cancer using the anti-GD2 ganglioside monoclonal antibody 3F8: a pilot trial. Eur J Nucl Med 23: 145-149.

12. Dobrenkov K, Cheung NK (2014) GD2-targeted immunotherapy and radioimmunotherapy. Semin Oncol 41: 589-612.

13. Kushner BH, Ostrovnaya I, Cheung IY, Kuk D, Kramer K, et al. (2015) Prolonged progression-free survival after consolidating second or later remissions of neuroblastoma with Anti-GD2 immunotherapy and isotretinoin: a prospective Phase II study. Oncoimmunology. 4: e1016704.

14. Horta ZP, Goldberg JL, Sondel PM (2016). Anti-GD2 mAbs and nextgeneration mAb-based agents for cancer therapy. Immunotherapy 8: 1097-1117.

15. Kircheis R, Halanek N, Koller I, Jost W, Schuster M, et al. (2012) Correlation of ADCC activity with cytokine release induced by the stably expressed, glyco-engineered humanized Lewis Y-specific monoclonal antibody MB314. MAbs 4: 532-541.

16. Chua JX, Vankemmelbeke M, Mcintosh RS, Clarke P, Moss R, et al. (2015) Monoclonal Antibodies Targeting LecLex-Related Glycans with Potent Antitumor Activity. Clin Cancer Res 21: 2963-2974.

17. Buskas T, Thompson P, Boons GJ (2009) Immunotherapy for cancer: synthetic carbohydrate-based vaccines. Chem Commun (Camb) 28: 5335-5349.

18. Guo Z, Wang Q (2009). Recent development in carbohydrate-based cancer vaccines. Curr Opin Chem Biol 13: 608-617.

19. Wilson RM, Danishefsky SJ (2013) A vision for vaccines built from fully synthetic tumor-associated antigens: from the laboratory to the clinic. J Am Chem Soc 135: 14462-14472.

20. Feng D, Shaikh AS, Wang F (2016) Recent Advance in Tumor-associated Carbohydrate Antigens (TACAs)-based Antitumor Vaccines. ACS Chem Biol 11: 850-863.

21. Ragupathi G, Koide F, Sathyan N, Kagan E, Spassova M, et al. (2003) A preclinical study comparing approaches for augmenting the immunogenicity of a heptavalent $\mathrm{KLH}$-conjugate vaccine against epithelial cancers. Cancer Immunol Immunother 52: 608-616.

22. Ragupathi G, Koide F, Livingston PO, Cho YS, Endo A, et al. (2006) Preparation and evaluation of unimolecular pentavalent and hexavalent 
Citation: Makhoul I, Monzavi-Karbassi B, Kieber-Emmons T (2016) Antibodies at the Center of Immunotherapy: Commentary on "Moving a Carbohydrate Mimetic Peptide into the Clinic". J Vaccines Vaccin 7: 343. doi:10.4172/2157-7560.1000343

Page 3 of 3

antigenic constructs targeting prostate and breast cancer: a synthetic route to anticancer vaccine candidates. J Am Chem Soc. 128: 2715-2725.

23. Sabbatini PJ, Ragupathi G, Hood C, Aghajanian CA, Juretzka M, et al. (2007) Pilot study of a heptavalent vaccine-keyhole limpet hemocyanin conjugate plus QS21 in patients with epithelial ovarian, fallopian tube, or peritoneal cancer. Clin Cancer Res 13: 4170-4177.

24. Pashov A, Monzavi-Karbassi B, Raghava G, Kieber-Emmons T (2007). Peptide mimotopes as prototypic templates of broad-spectrum surrogates of carbohydrate antigens for cancer vaccination. Crit Rev Immunol 27: 247-270.

25. Gabri MR, Cacciavillano W, Chantada GL, Alonso DF (2016) Racotumomab for treating lung cancer and pediatric refractory malignancies. Expert Opin Biol Ther 16: 573-578.

26. Kieber-Emmons T, Luo P, Qiu J, Chang TY, Magdalena OI, et al. (1999) Vaccination with carbohydrate peptide mimotopes promotes anti-tumor responses. Nat Biotechnol 17: 660-665.

27. Monzavi-Karbassi B, Artaud C, Jousheghany F, Hennings L, CarcelTrullols J, et al. (2005) Reduction of spontaneous metastases through induction of carbohydrate cross-reactive apoptotic antibodies. J Immunol 174: 7057-7065

28. Monzavi-Karbassi B, Hennings LJ, Artaud C, Liu T, Jousheghany F, et al. (2007) Preclinical studies of carbohydrate mimetic peptide vaccines for breast cancer and melanoma. Vaccine 25: 3022-3031.

29. Monzavi-Karbassi B, Luo P, Jousheghany F, Torres-Quiñones M, CuntoAmesty G, et al. (2004). A mimic of tumor rejection antigen-associated carbohydrates mediates an antitumor cellular response. Cancer Res 64: 2162-2166.

30. Wondimu A, Zhang T, Kieber-Emmons T, Gimotty P, Sproesser K, et al. (2008). Peptides mimicking GD2 ganglioside elicit cellular, humoral and tumor-protective immune responses in mice. Cancer Immunol Immunother 57: 1079-1089.

31. Pashov A, Monzavi-Karbassi, B, Kieber-Emmons T (2009) Immune surveillance and immunotherapy: lessons from carbohydrate mimotopes. Vaccine 27: 3405-3415.
32. Luo P, Canziani G, Cunto-Amesty G, Kieber-Emmons T (2000) A molecular basis for functional peptide mimicry of a carbohydrate antigen. J Biol Chem 275: 16146-16154.

33. Hennings L, Artaud C, Jousheghany F, Monzavi-Karbassi B, Pashov A, et al. (2011) Carbohydrate mimetic peptides augment carbohydrate-reactive immune responses in the absence of immune pathology. Cancers (Basel) 3: 4151-4169.

34. Hu J, Huang X, Ling CC, Bundle DR, Cheung NK (2009) Reducing epitope spread during affinity maturation of an anti-ganglioside GD2 antibody. J Immunol 183: 5748-5755.

35. Cheung NK, Lazarus H, Miraldi FD, Abramowsky CR, Kallick S, et al. (1987) Ganglioside GD2 specific monoclonal antibody 3F8: a phase I study in patients with neuroblastoma and malignant melanoma. J Clin Oncol 5: 1430-1440.

36. Murray JL, Cunningham JE, Brewer H, Mujoo K, Zukiwski AA, et al. (1994) Phase I trial of murine monoclonal antibody 14G2a administered by prolonged intravenous infusion in patients with neuroectodermal tumors. J Clin Oncol 12: 184-193.

37. Thurin J, Thurin M, Kimoto Y, Herlyn M, Lubeck MD, et al. (1987) Monoclonal antibody-defined correlations in melanoma between levels of GD2 and GD3 antigens and antibody-mediated cytotoxicity. Cancer Res 47: $1229-1233$.

38. Blaszczyk-Thurin M, Murali R, Westerink MA, Steplewski Z, Co MS, et al. (1996) Molecular recognition of the Lewis Y antigen by monoclonal antibodies. Protein Eng 9: 447-459.

39. Cheever MA, Allison JP, Ferris AS, Finn OJ, Hastings BM, et al. (2009) The prioritization of cancer antigens: a national cancer institute pilot project for the acceleration of translational research. Clin Cancer Res 15: 5323-5337.

40. Krengel U, Bousquet PA (2014) Molecular recognition of gangliosides and their potential for cancer immunotherapies. Front Immunol 5: 325. 